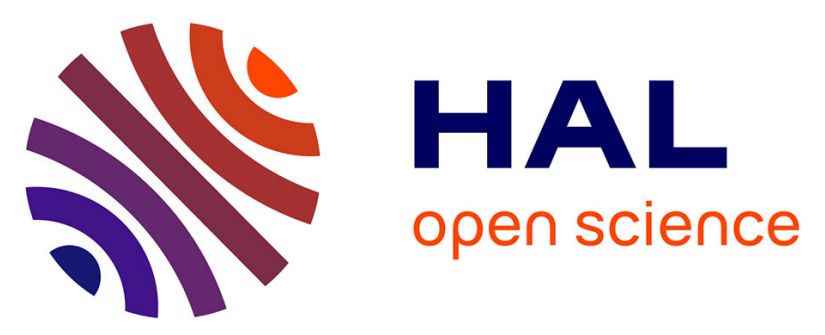

\title{
Compound cavity measurement of transmission and reflection of a tapered single-line photonic-crystal waveguide
}

\author{
Anne Talneau, Mikael Mulot, Srinivasan Anand, Philippe Lalanne
}

\section{To cite this version:}

Anne Talneau, Mikael Mulot, Srinivasan Anand, Philippe Lalanne. Compound cavity measurement of transmission and reflection of a tapered single-line photonic-crystal waveguide. Applied Physics Letters, 2003, 83 (16), pp.2577-2579. 10.1063/1.1568821 . hal-00874524

HAL Id: hal-00874524

https://hal-iogs.archives-ouvertes.fr/hal-00874524

Submitted on 18 Oct 2013

HAL is a multi-disciplinary open access archive for the deposit and dissemination of scientific research documents, whether they are published or not. The documents may come from teaching and research institutions in France or abroad, or from public or private research centers.
L'archive ouverte pluridisciplinaire HAL, est destinée au dépôt et à la diffusion de documents scientifiques de niveau recherche, publiés ou non, émanant des établissements d'enseignement et de recherche français ou étrangers, des laboratoires publics ou privés. 


\title{
Compound cavity measurement of transmission and reflection of a tapered single-line photonic-crystal waveguide
}

\author{
A. Talneau a) \\ CNRS/Laboratoire de Photonique et de Nanostructures, Route de Nozay, 91460 Marcoussis, France \\ M. Mulot and S. Anand \\ Royal Institute of Technology, Department of Microelectronics and Information Technology, \\ 164-40 Kista, Sweden \\ $\mathrm{Ph}$. Lalanne \\ Laboratoire Charles Fabry de l'Institut d'Optique, CNRS, Boile Postate 147,91403 Orsay, France
}

(Received 31 October 2002; accepted 21 February 2003)

\begin{abstract}
Measurements on a single-line defect photonic crystal waveguide demonstrate propagation losses as low as $140 \mathrm{~cm}^{-1}$ and coupling efficiency to a ridge access guide increased from $20 \%$ to $50 \%$ thanks to a tapered access. These results are obtained by analyzing the internal cavities created by residual reflections, hence, requiring a single sample. They are nevertheless crosschecked by measurements on distinct samples by the Fabry-Pérot resonance method. (C) 2003 American Institute of Physics.
\end{abstract}

[DOI: $10.1063 / 1.1568821]$

Photonic crystal (PC) guiding structures will result in compact and highly integrated photonic functions provided they can demonstrate low propagation losses and efficient coupling to classical guiding structures. Removing rows of holes in a regular two-dimensional (2D) PC creates a defect line which permits light propagation. A single line defect, named $W 1$, is an attractive candidate as it may support a single even mode for some wavelength domain. One disadvantage compared to broader PC guides is that the overlap of the field with the first inner line of holes is large, leading to propagation losses sensitive to any geometric change of the parameters. In order to check the losses for each fabrication run, one can use the Fabry-Pérot resonance method which has proved reliable for measuring propagation as well as transition losses, but requires numerous samples of variable lengths. The compound-cavity measurement presented here, based on the internal cavities due to residual reflections, allows to estimate on a single sample the propagation losses in the $W 1 \mathrm{PC}$ waveguide (PCW) and the PC/ridge taper efficiency. These results are also validated by the Fabry-Pérot resonance technique. Losses as low as $140 \mathrm{~cm}^{-1}$ are obtained on deeply etched $W 1 \mathrm{PCW}$ on a guiding heterostructure lying on InP substrate. This is comparable to the figure obtained in Ref. 1 for a membrane structure when the mode considered operates above the light cone, and highlights the potential of the "substrate" approach when ultimate fabrication quality is considered. ${ }^{2}$

Figure 1(a) presents the dispersion curve calculated by the plane wave expansion method of a $W 1 \mathrm{PCW}$, fabricated on a triangular 2D PC for a dielectric constant $\epsilon=10.56$, and an air filling factor $f=40 \%$. In the $1410-1590 \mathrm{~nm}$ wavelength domain investigated, the $W 1 \mathrm{PCW}$ supports a single even refractive mode. Figure 1(b) details the sample structure. For a reproducible coupling of the light in and out the $\mathrm{PC}$ section, the $W 1 \mathrm{PCW}$ is inserted in-between two ridge

${ }^{a)}$ Electronic mail: anne.talneau@1pn.cnrs.fr access guides also supporting a single even mode and limited by cleaved facets. ${ }^{3}$ A tapered section based on a gradual variation of holes size and depth ${ }^{4}$ is inserted at both ridge/ $W 1$ transitions to match the modal shape of the field in the ridge access guide to that of the strongly confined $W 1 \mathrm{PCW}$ [Fig. 2(a)]. PCWs are fabricated on a two-dimentional PC consisting of a triangular array (period $a=450 \mathrm{~nm}$ ) of holes drilled on a 500-nm-thick GaInAsP confining layer. These holes are etched by chemically assisted ion beam etching (CAIBE) using $\mathrm{Ar} / \mathrm{C} 12$ chemistry. ${ }^{5} \mathrm{~A}$ micrograph of the etched holes shows in Fig. 2(b) holes deeper than $3 \mu \mathrm{m}$ for an air-filling factor $f=40 \%$. The first hole of the taper section is (somehow by chance) visible on the left side.

The Fabry-Pérot resonance method, previously used for wide PC guides, ${ }^{3}$ allows accurate measurement of the propagation and transition losses, but requires several samples of variable PC lengths. As a reference to validate the compound-cavity measurement, we carried out the earlier method on samples with three different lengths for the $W 1$ PC section: 120, 180, and 240 rows. Each sample has a ten row long taper which gradually reduces the PCW from a three-missing-line wide PCW to a $W 1 \mathrm{PCW}$, as described in Ref. 4, see Fig. 2(a). Measurements are performed in TE polarization, on a $1410-1590 \mathrm{~nm}$ wavelength span covered
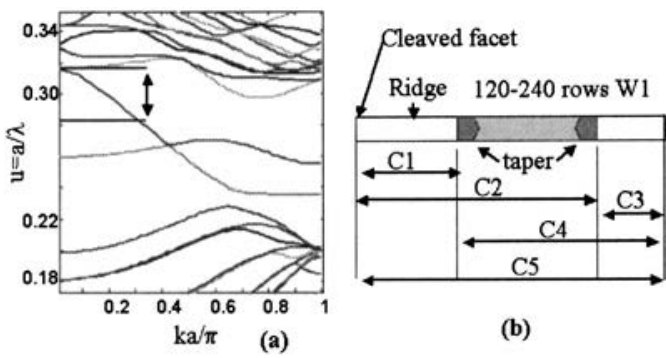

FIG. 1. (a) W1 PCW dispersion curve, the investigated wavelength domain is shown (b) sketch of the sample structure showing the different internal cavities. 


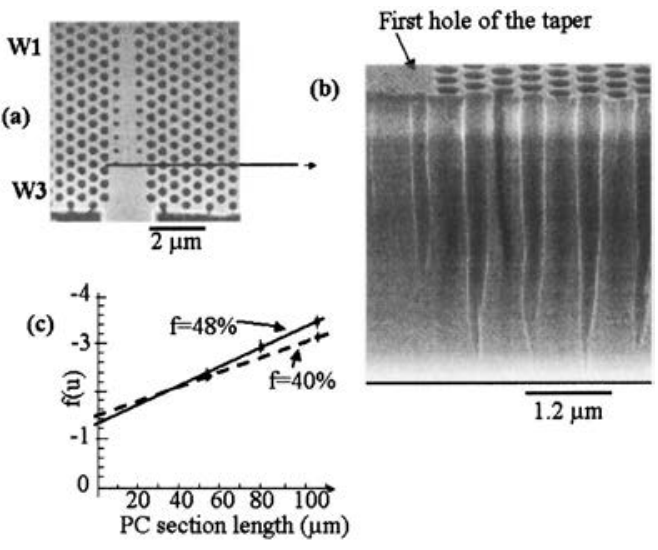

FIG. 2. Micrographs of (a) top view of CAIBE etched taper, (b) cross section of $W 1 \mathrm{PCW}$, (c) propagation losses in the $W 1-\mathrm{PCW}$.

by two tunable laser sources. At variance with Ref. 3, the residual reflection of the taper remains sizeable, leading to multiple cavity signature in the Fourier transformed spectrum. On the transmitted spectrum, we Fourier filtered systematically the peak corresponding to the total cavity $C 5$ unambiguously identified from its physical length. This leads to the fringe contrast $u=\left(P_{\min } / P_{\max }\right)^{1 / 2}$ of cavity $C 5$ which is related to the losses in the $W 1$ denoted $\alpha_{\mathrm{pc}}$, and to the transmission at the taper level $t_{\text {tap }}$ through the Fabry-Pérot resonator relation (1):

$\operatorname{Ln}\left(r_{c}^{2}\right)-\alpha_{r} L_{r}-\alpha_{\mathrm{pc}} L_{\mathrm{pc}}+4 \operatorname{Ln}\left(t_{\mathrm{tap}}\right)=\operatorname{Ln}[(1-u) /(1+u)]$.

This treatment is performed on limited spectral windows in order to reduce the Fourier peaks smearing arising from index dispersion. The ridge is calibrated: $r_{c}=0.6$ is the reflection coefficient of the cleaved facets, and $\alpha_{r}=3.5 \mathrm{~cm}^{-1}$ are the propagation losses in the ridges acces guides.

We then plot on Fig. 2(c) $f(u)$ versus the $W 1$ PCW length $L_{\mathrm{pc}}$ :

$$
f(u)=\operatorname{Ln}[(1-u) /(1+u)]-\left[\operatorname{Ln}\left(r_{c}^{2}\right)-\alpha_{r} L_{r}\right],
$$

$u$ being the fringe contrast in the $1500-1520 \mathrm{~nm}$ wavelength domain, for two air-filling factors: $f=48 \%$ and $f=40 \%$. From the slope of $f(u)$, we calculate $\alpha_{\mathrm{pc}}$, and from the intersect at $L_{\mathrm{pc}}=0$, we obtain $t_{\text {tap }}$. For $f=48 \%$, losses are $180 \mathrm{~cm}^{-1}$, and the power coupled through the taper from the ridge to the $W 1 \mathrm{PCW}$ is $T=\left(t_{\text {tap }}\right)^{2}=51.8 \%$; for $f=40 \%$, losses are reduced to $140 \mathrm{~cm}^{-1}$, and the power coupled is $T=46.2 \%$. Due to the large overlap of the optical field with the holes in the case of $W 1 \mathrm{PCW}$, these propagation losses are fairly sensitive to the air-filling factor of the 2D PC, whereas $t_{\text {tap }}$ is not. Even if these losses are quite low for a $W 1 \mathrm{PCW}$, still, we cannot claim that the present level is the "intrinsic" one for infinite perfect holes. ${ }^{2}$

The taper efficiency is found to be limited to $50 \%$. As seen on the micrograph of Fig. 2(b), the first hole of the taper is $180 \mathrm{~nm}$ in diameter and about $2.7 \mu \mathrm{m}$ deep. The hole size is clearly much larger than what is required for perfect matching structure as calculated in Ref. 6 with very small holes. The taper efficiency is weakly dependent on the airfilling factor, but depends mainly on the sizes of the successive gradual holes, especially the size of the smallest one.

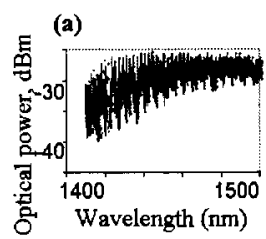

(c)

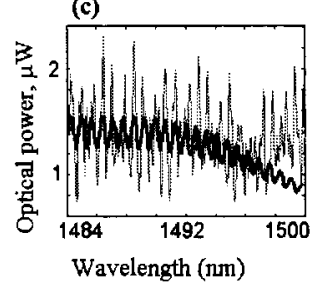

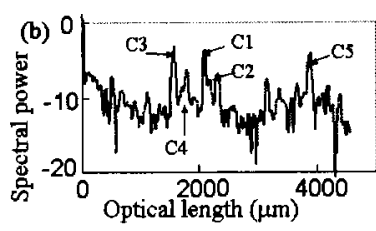

(d)

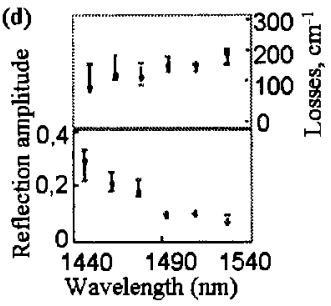

FIG. 3. 120 rows long $W 1-\mathrm{PCW}$ with taper access: (a) transmitted power spectrum, (b) spectral power on the 1484-1500 nm window, (c) unfiltered (thin line) and filtered (bold line) transmitted power with a filter suited to $C 1$ cavity, and (d) taper reflection and propagation losses as a function of wavelength.

Refering to Fig. 1(b), one can take advantage of the internal cavities generated by the residual reflection at the tapered ridge/ $W 1 \mathrm{PCW}$ transitions. These cavities denoted from $C 1$ to $C 5$ are visible as peaks when Fourier transforming the transmitted spectrum. The compound-cavity measurement is based on the identification of each internal cavity whose physical length is carefully measured on the sample itself, and whose contribution is clearly visible when Fourier transforming the transmitted spectrum. This spectrum is filtered on a chosen cavity, and the corresponding fringe contrast is related to the propagation losses and reflection/ transmission at the different interfaces, as expressed in Eq. (1). One can thus measure on a single sample both the reflection at the tapered access and the propagation losses. Let us consider here a sample as described in Fig. 1(b) with 120 rows of $W 1$ PCW, $f=40 \%$ and a taper on both sides; the physical lengths of the cavities are measured with a microscope: $C 1=545 \mu \mathrm{m} ; C 2=599 \mu \mathrm{m} ; C 3=415 \mu \mathrm{m} ; C 4=469$ $\mu \mathrm{m}$; and $C 5=1014 \mu \mathrm{m}$. Figure 3 shows the transmission spectrum (a) and the Fourier transformed spectrum (b) calculated on limited spectral windows, only the 1484-1500 $\mathrm{nm}$ window is plotted. Using limited spectral windows reduces the peak smearing due to mode index dispersion which translates into a better accuracy of the peak associated optical length. On Fig. 3(b), one can identify the different physical cavities $C 1-C 5$. With the ridge calibrated (yielding $r_{c}$ and $\alpha_{r}$ ), the earlier exercise is first carried out for the fringe contrast $u$ of cavity $C 1$ for six adjacent spectral windows. Figure 3(c) presents the unfiltered (thin line) and filtered (bold line) transmitted powers on the restricted 1484-1500 $\mathrm{nm}$ domain for $C 1$ cavity. The constrat $u$ is measured on the bold filtered curve

$$
\left.\operatorname{Ln}\left(r_{c}^{*} r_{\text {tap }}\right)\right)-\alpha_{r} L_{r}=\operatorname{Ln}[(1-u) /(1+u)], \quad \text { cavity } C 1 .
$$

The result is the reflectivity spectrum of the taper $r_{\text {tap }}$ plotted in Fig. 3(d). For the 1540-1590 nm wavelength domain, the peak associated to cavity $C 1$ is no more visible, indicating that the reflection amplitude is below 0.1 . This residual reflection comes from the taper fabrication as previously explained. The spectral behavior of the taper will be discussed elsewhere, but one can readily notice that for a large wave- 
length range $(1500-1590 \mathrm{~nm})$, the taper reflection coefficient is below $1 \%$. One can also notice that the accuracy on $r_{\text {tap }}$ is reduced in the domain where $r_{\text {tap }}$ is large $(>0.15)$, due to some coupling of the internal cavities.

The fringe contrast $u$ of cavity $C 2$ is related to the losses in the $W 1 \mathrm{PC}$ section $\alpha_{\mathrm{pc}}$, the tapered transition $t_{\mathrm{tap}}=0.7$ and $r_{\text {tap }}$ just evaluated earlier, according to Eq. (4):

$$
\begin{aligned}
& \operatorname{Ln}\left(r_{c}^{*} r_{\mathrm{tap}}\right)-\alpha_{r} L_{r}-\alpha_{\mathrm{pc}} L_{\mathrm{pc}}+2 \operatorname{Ln}\left(t_{\mathrm{tap}}\right) \\
& \quad=\operatorname{Ln}[(1-u) /(1+u)], \quad \text { cavity } C 2 .
\end{aligned}
$$

When carrying out a similar exercise for the $C 2$ cavity, one obtains the spectral dependence of the propagation losses in the $W 1120$ rows long section, as plotted in Fig. 3(d). Again, for $\lambda>1520 \mathrm{~nm}$, the $C 2$ peak is no more visible. Losses are in the (150-200 $\left.\mathrm{cm}^{-1}\right)$ range, nearly constant on a large wavelength span. This value is in good agreement with the one accurately determined by the Fabry-Pérot method, and required indeed a single sample. The reduced propagation losses measured when the wavelength is decreased could be due to the fact that one approaches the band edge [see Fig. 1(a) and Ref. 7].

In order to estimate the taper efficiency, we fabricated the same structure as in Fig. 1(b), but ridge/W1 PCW transitions are now abrupt, without any taper. The same procedure on cavity $C 1$ led to a reflection coefficient for the abrupt transition $r_{\text {abr }}=0.25$, nearly independent of the wavelength for the spectral interval considered, which seems reasonable. The fringe contrast of cavity $C 2$ is then used to estimate the abrupt transmission amplitude coefficient $t_{\mathrm{abr}}$, as the losses within the $W 1 \mathrm{PC}$ section are known. We obtain
$T=20 \%$. When compared to $T=50 \%$ in the case of the tapered access, the improvement is larger than a factor 2. As previously explained, a better fabrication requiring the manufacture of smaller holes would further increase the taper efficiency.

The method presented here takes advantage of residual internal reflection in a compound-cavity structure to measure on a single sample both interface reflection and propagation losses. Reflection as low as $1 \%$ leads to peaks that can be clearly identified in the Fourier transformed spectrum. Reduced spectral windows increase the peaks visibility. This method has allowed to measure propagation losses as low as $140 \mathrm{~cm}^{-1}$ on a $W 1 \mathrm{PCW}$, and an increased transmission from $20 \%$ to $50 \%$ for a taper design based on a gradual variation of holes size.

This work is supported by the european IST PCIC project. M.M. and S.A. acknowledge the Swedish Strategic Research Foundation for partially supporting this work.

${ }^{1}$ T. Baba, A. Monegi, T. Iwai, N. Fukaya, Y. Watanabe, and A. Sakai, IEEE J. Quantum Electron. 38, 743 (2002).

${ }^{2}$ H. Benisty, Ph. Lalanne, S. Olivier, M. Rattier, C. Weisbuch, C. J. M. Smith, T. F. Krauss, C. Jouanin, and D. Cassagne, Opt. Quantum Electron. 34, 205 (2002).

${ }^{3}$ A. Talneau, L. Legouezigou, and N. Bouadama, Opt. Lett. 26, 1259 (2001).

${ }^{4}$ A. Talneau, Ph. Lalanne, M. Agio, and C. M. Soukoulis, Opt. Lett. 27, 1522 (2002).

${ }^{5}$ M. Mulot, S. Anand, C. F. Carlstrom, M. Swillo, and A. Talneau, Phys. Scr., T T101, 106 (2002).

${ }^{6} \mathrm{Ph}$. Lalanne and A. Talneau, Opt. Express 10, 354 (2002).

${ }^{7}$ M. Loncar, D. Nedeljkovic, T. P. Pearsall, J. Vuckovic, A. Scherer, S. Kuchinsky, and D. C. Allan, Appl. Phys. Lett. 80, 1689 (2002). 